\section{Check for updates}

Cite this: Soft Matter, 2018, 14,7246

Received 18th January 2018, Accepted 5th August 2018

DOI: 10.1039/c8sm00133b

rsc.li/soft-matter-journal

\title{
DMA study of water's glass transition in nanoscale confinement
}

\author{
V. Soprunyuk and W. Schranz (D)*
}

\begin{abstract}
Dynamic mechanical analysis (DMA) measurements of water confined in nanoporous silica have been performed as a function of temperature and frequency for different pore sizes $(2.5-10 \mathrm{~nm})$ at heating and cooling. Most of the data show three processes, $P_{1}, P_{2}$ and $P_{3}$, where $P_{1}$ and $P_{2}$ depend on measurement frequency and $P_{3}$ does not. The characteristic shift of $P_{3}$ with pore size shows that this process corresponds to freezing/melting of "internal water", i.e. in the core of the pores. Thermal expansion data indicate - in agreement with e.g. [A. Taschin, P. Bartolini and R. Torre, Meas. Sci. Technol., 2017, 28, 014009] - that in all our nanoporous systems about 2 layers of water remain liquid much below the freezing point. Dynamic elastic measurements show clear signatures of glass freezing of this supercooled water in the vicinity of P1. Extrapolating the DMA data to the timescale $\left(10^{3} \mathrm{~s}\right)$ of adiabatic calorimetry unveils a systematic behaviour: $P_{1}(T)$ shows a clear size dependence for a broad range of pore diameters, i.e. $2.5 \mathrm{~nm} \leq d \leq 52 \mathrm{~nm}$, implying (together with the corresponding activation energy $0.5 \mathrm{eV}$ ) that $\mathrm{P}_{1}$ corresponds to the glass-liquid transition of a few layers of supercooled water at $T_{\mathrm{g}}(d)$. An extrapolation of $T_{\mathrm{g}}(d)$ to $d \rightarrow \infty$ yields $T_{\mathrm{g}}(\infty) \approx 136 \mathrm{~K}$, the traditional value for bulk water. The small (liquid like) value of Young's modulus in a temperature region above P1 is most naturally explained assuming that the supercooled water in this range is still liquid, implying that $T_{\mathrm{g}}$ values of $160 \mathrm{~K}$ or even $210 \mathrm{~K}-$ as suggested by various authors - are unlikely.
\end{abstract}

\section{Introduction}

Water is of fundamental importance for all living organisms as well as for abiotic environments. Despite its simple molecular structure when compared with other glass forming liquids, some important thermodynamic and kinetic properties are still rather controversially discussed. ${ }^{1}$ A vexed question relates e.g. to the location of its bulk glass transition $T_{\mathrm{g}}$. In vapour-deposited amorphous solid water $(\mathrm{ASW})^{2}$ as well as in rapidly quenched water below $100 \mathrm{~K}$ (hyperquenched glassy water HGW) ${ }^{3,4}$ a glass to liquid transition was reported from calorimetric measurements to occur at $T_{\mathrm{g}}=136 \mathrm{~K}$, which then was assigned to the bulk glass transition temperature of water.

Later, Angell et al. ${ }^{5,6}$ proposed a much higher value of bulk $T_{\mathrm{g}} \approx 165 \mathrm{~K}$, based on the heat-release of HGW.

Unfortunately, the glass transition of water cannot be easily studied, since water can only be supercooled at most down to $235 \mathrm{~K}$, the homogeneous nucleation temperature, below which it crystallizes. A common method to avoid crystallization is to put water into nanoscale confinement. E.g. Cerveny et al. ${ }^{7}$ studied water confined in vermiculite clay and bread by dielectric and DSC measurements, arriving at the conclusion that $T_{\mathrm{g}} \approx 160-165 \mathrm{~K}$.

University of Vienna, Faculty of Physics, Physics of Functional Materials, Boltzmanngasse 5, A-1090 Wien, Austria.E-mail: wilfried.schranz@univie.ac.at
Oguni et al..$^{8-11}$ studied the glass transition of water in various nanoscale confinements (silica-gel nanopores with rather irregular pores and MCM-41 with regular pore structures) by adiabatic calorimetry, suggesting ${ }^{11}$ a value of $T_{\mathrm{g}}=210 \mathrm{~K}$ for bulk water.

Despite all efforts, even after decades of research ${ }^{12,13}$ many properties of water are still heavily discussed. An important dispute relates to the question of a possible "fragile-strong", transition, triggered by an observed crossover from superArrhenius to Arrhenius behaviour around $T_{\mathrm{FS}} \approx 225 \mathrm{~K}$. For bulk supercooled water a "fragile to strong" (FS) transition was proposed at $T_{\mathrm{FS}} \approx 228 \mathrm{~K}^{\mathbf{1 4 , 1 5}}$ based on dielectric relaxation. A similar dynamic crossover was also found ${ }^{16-18}$ by different experimental techniques for confined supercooled water and there is an ongoing discussion ${ }^{1}$ about the origin of such a crossover.

A possible scenario relates the FS-crossover ${ }^{19,20}$ to a transition ${ }^{21}$ (liquid-liquid critical point) from a high density liquid (HDL at $T>T_{\mathrm{FS}}$ ) to a low density liquid (LDL at $T<T_{\mathrm{FS}}$ ). Another interpretation $^{17}$ assumes that the dynamic crossover of confined water is due to a confinement induced crossover from a cooperative $\alpha$-process to a Johari-Goldstein type local $\beta$-process. A possible explanation for such a change is that at the crossover temperature - observed in dielectric data at about $180 \mathrm{~K}$ - the characteristic length $\xi$ of dynamically correlated regions becomes 
equal to the size $d$ of the confinement, which limits the characteristic length at a constant value $\xi\left(T_{\mathrm{FS}}\right)=d$ and as a result the high-temperature super-Arrhenius dependence (Vogel-Fulcher, power-law) of $\tau_{\alpha}$ transforms to a low-temperature Arrhenius behavior $\tau_{\beta}$. This scenario would imply that the dynamic correlation length $\xi$ of water should be in the range of $2 \mathrm{~nm}$ around $T_{\mathrm{FS}} \approx 180 \mathrm{~K}$.

In another approach some authors show ${ }^{22,23}$ that a powerlaw $\sim\left(T-T_{x}\right)^{-\gamma}$ fits the observed super-Arrhenius behaviour of viscosity $\eta$ or relaxation time $\tau$ better than a Vogel-Fulcher law. Such a power-law can be interpreted in several ways. One is mode-coupling theory, ${ }^{24}$ where $T_{x}=T_{\mathrm{c}}>T_{\mathrm{g}}$ is the modecoupling temperature. Another explanation ${ }^{25,26}$ suspects the existence of an order-disorder transition around $T_{x} \approx 225 \mathrm{~K}$, which however cannot be reached due to prior crystallization. The power-law would then occur due to the appearance of precursor fluctuations, which would lead to a $\lambda$-type anomaly in the specific heat at $T_{x}$. Using the Adam-Gibbs equation ${ }^{27}$ $\tau=\tau_{0} \exp \left(C / T S_{\mathrm{c}}\right)$, the strong-fragile transition would then be a direct consequence of the corresponding configurational entropy change $S_{\mathrm{c}}$.

An idea which then was further perpetuated ${ }^{26}$ is that the glass transition in water around $136 \mathrm{~K}$ is nothing else than an ergodicity breaking transition (i.e. due to freezing of some remaining orientational degrees of freedom) appearing in the tail of the order-disorder transition, quite similar to that demonstrated $^{28,29}$ e.g. for $\mathrm{C}_{60}$.

Another idea, which is quite consistent with the previous one, was recently proposed ${ }^{30}$ on the basis of a study of the reorientation dynamics of water molecules in different ice phases. There the authors claim that the calorimetric signatures of the amorphous ices (around $136 \mathrm{~K}$ for LDA) that have been interpreted as a glass-to-liquid transition, occur due to unfreezing of re-orientation of water molecules and not due to unfreezing of long range molecular diffusion. The real glass transition corresponding to unfreezing of translational diffusion of water molecules - would then occur at higher temperature, which the authors preliminarily associate with the fragile-strong transition at $T_{\mathrm{FS}}$.

Unfortunately we cannot measure the crossover from Arrhenius to super-Arrhenius behaviour with our DMA method, since we are restricted in frequency to at most $100 \mathrm{~Hz}$. But from the value of the activation energy of the order of $E_{\mathrm{a}} \approx 0.5 \mathrm{eV}$ (see below), which is in very good agreement with previous dielectric data, ${ }^{1,7,31}$ we can conclude that one of our damping peaks (P1) corresponds to the same process as the denoted $\beta$-process.

Below we will show that we have strong evidence that this damping peak (P1) is not a local process, but originates from a glass-to-liquid transition of water in the nanopores. We mainly infer this from the fact that we observe a very pronounced softening in the real part of the complex Young's modulus, which starts in the temperature region of the damping peak P1 and extends to temperatures near the proposed $T_{\mathrm{FS}}$.

Although not so commonly used, dynamic mechanical analysis (DMA) turned out to be an excellent method ${ }^{32-34}$ for the study of confined glass forming liquids.
In the present work we show (Section 3) results of dynamic mechanical analysis (DMA) and thermomechanical (TMA) measurements of water confined in nanoporous silica, Vycor and Gelsil with pore diameters $d=10 \mathrm{~nm}$ (V10), $5 \mathrm{~nm}$ (G5) and $2.5 \mathrm{~nm}$ (G2), respectively, as a function of temperature (heating and cooling) and frequency $(0.1-70 \mathrm{~Hz})$.

Combining our data with adiabatic calorimetry data of Oguni et al. ${ }^{8-11}$ unveils a clear systematic behaviour concerning the thermal relaxation processes of water in nanopores over a pore size range of $d=1.2-52 \mathrm{~nm}$, which is presented also in Section 3. In Section 2 we specify the host materials which are used to confine water and the DMA-method is briefly described. Section 4 concludes the paper.

\section{Experimental}

Table 1 summarizes the main specifications of the $\mathrm{SiO}_{2}$-based nanoporous host materials, as obtained ${ }^{35-37}$ from $\mathrm{N}_{2}$ adsorption and SAXS measurements.

Vycor (Corning Inc., New York), is produced via phase separation within a $\mathrm{Na}_{2} \mathrm{O}-\mathrm{B}_{2} \mathrm{O}_{3}-\mathrm{SiO}_{2}$ melt, and subsequent acid leaching, which leaves a $96 \%$ pure $\mathrm{SiO}_{2}$ skeleton containing interconnected cylindrical pores of random length and direction. A narrow pore size distribution and an average ratio of pore length $l$ over pore diameter $d$ of $l / d \approx 4$ is reported. ${ }^{37}$ Gelsil samples result from a sol-gel process ${ }^{38}$ and consist of randomly formed pure fused $\mathrm{SiO}_{2}$ monodisperse spheres ${ }^{39}$ touching and penetrating each other. The voids between these spheres create a random network of inter-connected corridors and pockets and show a larger pore size distribution as compared to Vycor.

For DMA measurements macroscopic samples of a few $\mathrm{mm}^{3}$ are needed. A diamond saw was used to cut the samples of Vycor and Gelsil with typical sizes of $3 \times 2 \times 2 \mathrm{~mm}^{3}$. The samples were sanded to gain parallel surface plains. The geometrical accuracy of the polished samples was better than $10 \mu \mathrm{m}$. Then the samples were cleaned by first dropping them into a $30 \% \mathrm{H}_{2} \mathrm{O}_{2}$ solution at $90{ }^{\circ} \mathrm{C}$ for $24 \mathrm{~h}$, followed by drying at $120{ }^{\circ} \mathrm{C}$ in a high vacuum chamber also for $24 \mathrm{~h}$. Filling of the samples with distilled water was done by spontaneous imbibition. ${ }^{40}$

With the effective volume of a water molecule in the liquid state $\left(\mathrm{ca} .3 \times 10^{-29} \mathrm{~m}^{3}\right)$, a rough estimation yields a number of about 300 water molecules in $2.5 \mathrm{~nm}$ pores, 2400 in $5 \mathrm{~nm}$ pores and about 100000 water molecules in Vycor with $10 \mathrm{~nm}$ pores.

For thermal expansion measurements we used a TMA 4000 (Perkin Elmer) as well as a Diamond DMA (Perkin Elmer).

To study the slow dynamics of confined supercooled water, we performed Dynamic Mechanical Analysis (DMA) measurements

Table 1 Characteristic parameters ${ }^{35-37}$ of nanoporous silica

\begin{tabular}{llll}
\hline Properties & V10 & G5 & G2 \\
\hline Pore size $(\mathrm{nm})$ & $10 \pm 0.5$ & $5 \pm 0.8$ & $2.5 \pm 1$ \\
Porosity & 0.4 & 0.54 & 0.36 \\
Pore surface area/pore volume & 4.5 & 8.3 & 15 \\
Pore surface area $\left(\mathrm{m}^{2} \mathrm{~g}^{-1}\right)$ & 90 & 510 & 590 \\
Surface fractional dimension $D_{\mathrm{s}}$ & 2.3 & 2.24 & -
\end{tabular}


using two devices (DMA 8000 and Diamond DMA, Perkin Elmer). With DMA, the real $Y^{\prime}$ and imaginary $Y^{\prime \prime}$ parts of the complex Young's modulus $Y^{*}=Y^{\prime}+\mathrm{i} Y^{\prime \prime}$ are determined from the measured sample strain and phase shift between the externally applied dynamic force and the sample strain. A force up to $10 \mathrm{~N}$ can be applied, with a resolution of $0.002 \mathrm{~N}$. The resolution of the sample height is about $3 \mathrm{~nm}$ and the phase shift $\delta$ can be measured with an accuracy of about $0.1^{\circ}$. The relative accuracy of the DMA method is about $0.2-1 \%$, but the absolute accuracy of such a measurement is usually not better than $20 \%$. To obtain reasonable absolute values we have normalized our measured $Y^{\prime}$-data at room temperature to the Young's modulus data previously measured ${ }^{35}$ by RUS (resonance ultrasonic spectroscopy). The $Y^{\prime \prime}$ values are then obtained from the phase shift data $\delta$ using $Y^{\prime \prime}=Y^{\prime} \tan \delta$.

The measurement frequency can be varied between $0.01 \mathrm{~Hz}$ and $100 \mathrm{~Hz}$ at temperatures between $80 \mathrm{~K}$ and $600 \mathrm{~K}$. More details of the DMA method are given in ref. 41 and 42 .

Here we present DMA and TMA results of water in Gelsil (2.5 nm, G2 and $5 \mathrm{~nm}, \mathrm{G} 5)$ and Vycor (10 nm, V10) (Table 1) measured at cooling and heating as a function of temperature and frequency.

Filling is done by making use of the strong capillary forces. To avoid the formation of air bubbles the sample should be attached to water only from one side. After full filling (controlled by a balance) the samples become transparent.

\section{Results and discussion}

\section{Thermal expansion of water confined in nanopores}

Very recently, we have measured ${ }^{43}$ the mechanical relaxation behaviour of supercooled water in Vycor and Gelsil by quenching the samples down to $80 \mathrm{~K}$, followed by subsequent heating at a rate of $1.5 \mathrm{~K} \mathrm{~min}^{-1}$. In the present work we use rather moderate cooling/heating rates of $2 \mathrm{~K} \mathrm{~min}^{-1}$.

It is instructive to compare the data from quenching experiments with the present ones. As an example we show (Fig. 1) the thermal expansion behaviour of water in G2 for quenched samples and slowly cooled/heated $\left(2 \mathrm{~K} \mathrm{~min}^{-1}\right)$ samples. A very similar behaviour was also found for water confined in G5 and V10. It is evident that even in $2.5 \mathrm{~nm}$ pores some portion of ice is formed, most probably in the central part of the pores (see below). After quenching to about $80 \mathrm{~K}$, the formation of ice takes a few minutes as can be seen in the inset of Fig. 1.

The big difference between quenched and slowly cooled samples in the magnitude of the anomalies suggests that in quenched samples a larger portion of water freezes to ice compared to the slowly cooled samples. A similar cooling/heating rate dependence of thermal expansion of water confined in Vycor (Corning no. 7930, pore diameter $c a .6 \mathrm{~nm}$ ) was reported ${ }^{44}$ much earlier. The authors explained this time dependent expansion by a transport process inside the pores, which is needed to dissipate pressure generated by the ice formation. The pressure distribution of water confined in cylindrical pores of MCM41 was recently studied by molecular dynamics simulations ${ }^{45}$ in a

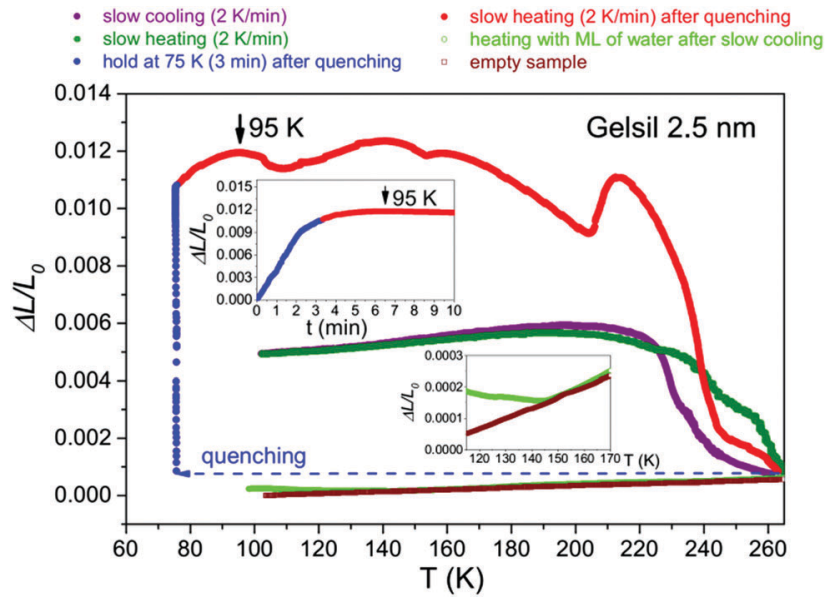

Fig. 1 Thermal expansion of water in Gelsil G2 for quenched (red) and slowly cooled/heated ( $2 \mathrm{~K} \mathrm{~min}^{-1}$, green/violet) samples compared with partially filled Gelsil (light green) and empty Gelsil (brown).

temperature range from $298 \mathrm{~K}$ to $210 \mathrm{~K}$. The author found that in this range water is under significant negative pressure. But the presence of ice in the core of the pores renders the situation much more complex and at present we cannot draw a final conclusion on the observed time dependence of thermal expansion and Young's moduli data.

In our recent work ${ }^{43}$ we used thermal expansion data of water confined in nanoporous silica measured at $2 \mathrm{~K} \mathrm{~min}^{-1}$ rates to estimate the relative amount of supercooled $\left(V_{\mathrm{sw}}\right)$ water for various pore sizes below the freezing point, yielding $V_{\text {sw }} / V_{\text {pore }} \approx 0.6,0.3$ and 0.1 for G2, G5 and V10, respectively.

If - in correspondence to a recent study ${ }^{46}$ of water in Vycor (4 nm pore size) - we assume that two layers of water remain liquid below the freezing temperature, and approximating the complex cavities of the present nanoporous silica matrices (Vycor and Gelsil) by cylindrical pores and the water molecules with a diameter of about $0.3 \mathrm{~nm}$, we obtain $V_{\text {sw }} / V_{\text {pore }} \approx 0.7,0.4$ and 0.2 for G2, G5 and V10, respectively. Although these estimations are rather rough, they show that it is reasonable to assume that a few layers of supercooled water can take part in the glass freezing process of water in the presently used nanoporous silica. This is also in agreement with recent proton NMR spectroscopy ${ }^{47}$ of water confined in 1-10 nm sized pores.

\section{Dynamic mechanical response of water in nanopores}

In addition to thermal expansion, DMA measurements yield information about dynamical processes of supercooled water in the nanoporous silica host materials.

Fig. 2 shows $Y^{\prime}$ and $Y^{\prime \prime}$ for water in Vycor and Gelsil for heating after cooling at $2 \mathrm{~K} \mathrm{~min}^{-1}$.

Three peaks are visible in $Y^{\prime \prime}$, denoted as P1, P2 and P3. The general behaviour is in excellent agreement with earlier mechanical relaxation measurements ${ }^{44}$ of water in Vycor $(7930, d=6 \mathrm{~nm}$ ), measured at about $400 \mathrm{~Hz}$. Inspecting the temperature variation of $Y^{\prime}$ in Fig. 2 one observes that at about $100 \mathrm{~K}$ the Young's modulus has increased by more than $70 \%$ over the room temperature value (see also Fig. 3-5), whereas at about $200 \mathrm{~K}$ - where a large fraction of 

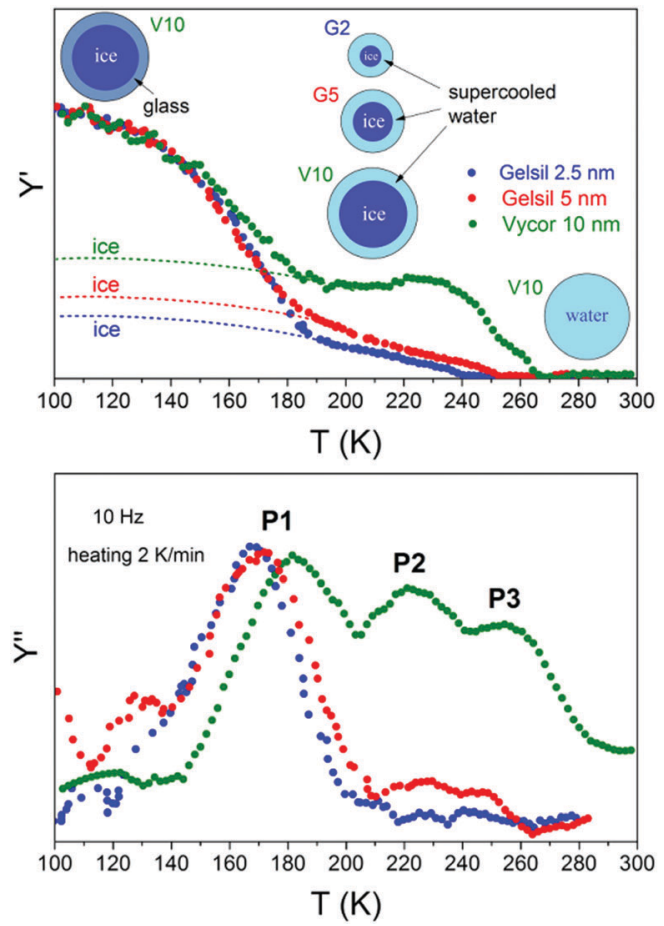

Fig. 2 Real $Y^{\prime}$ and imaginary $Y^{\prime \prime}$ parts of the complex Young's modulus of water in Gelsil $2.5 \mathrm{~nm}, 5 \mathrm{~nm}$ and Vycor $10 \mathrm{~nm}$, measured at a heating rate of $2 \mathrm{~K} \mathrm{~min}^{-1}$ after cooling at a cooling rate of $2 \mathrm{~K} \mathrm{~min}^{-1}$. The inset shows a sketch of the different states of water according to the presumed core-shell model. ${ }^{46}$

water has frozen $-Y^{\prime}$ has only increased by some $10 \%$. This is another signature of the effect of the liquid film of supercooled water that surrounds the ice core in the pores and decouples (with respect to elasticity) the ice from the silicon matrix.

To get additional information on the dynamic processes of supercooled water in the pores we performed measurements at various frequencies. Fig. 3 shows the data for water in Gelsil $2.5 \mathrm{~nm}$ at frequencies between 0.1 and $70 \mathrm{~Hz}$. Interestingly enough, the re-hardening process which was observed ${ }^{43}$ in the quenched samples prior to melting is strongly suppressed (for V10) or even absent (for G5 and G2) in slowly cooled samples. This has the advantage that the elastic softening in the vicinity of P1 becomes now more clearly visible, since it is no longer obscured by the re-hardening process of water in quenched samples. Similar to the quenched samples, ${ }^{43}$ the low temperature peak P1 shifts to higher temperatures with increasing frequency.

Before we discuss different possible origins of the relaxation peak P1 in more detail, let us see which relaxation function fits the data well. Following previous dielectric measurements of confined water ${ }^{7,48}$ we use a symmetric Cole-Cole (C-C) function

$$
Y^{*}(\omega)=Y_{\infty}-\frac{\Delta Y}{1+(i \omega \tau)^{\alpha}}
$$

with $\tau_{\mathrm{P}_{1}}=\tau_{01} \exp \left(E_{\mathrm{a}} / k_{\mathrm{B}} T\right)$.

It turns out that the Cole-Cole relaxation (1) fits the data as well as it can be expected. The real part of the complex Young's modulus is nearly perfectly described in a broad temperature range around P1. For the imaginary part deviations below and
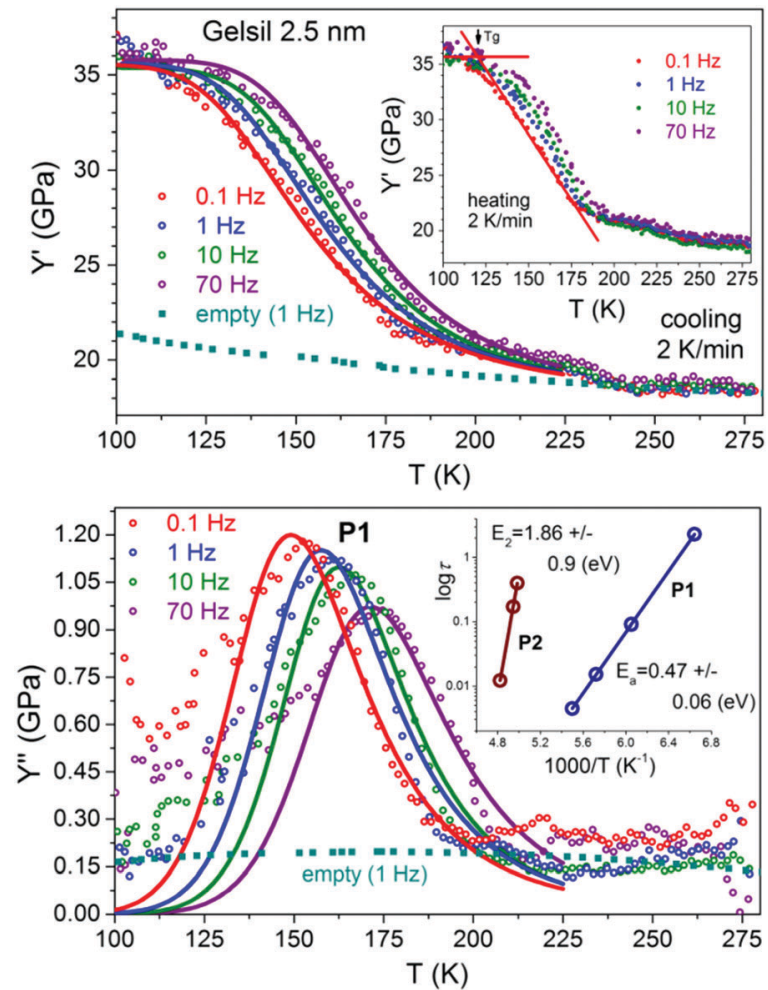

Fig. 3 Temperature dependencies of $Y^{\prime}$ and $Y^{\prime \prime}$ of slowly cooled water in Gelsil $2.5 \mathrm{~nm}$ at different frequencies. The blue squares show results of empty samples. Insets show a corresponding heating run and an Arrhenius plot corresponding to $\mathrm{P} 1$ and $\mathrm{P} 2$. The activation energy $E_{2}$ is determined from DMA measurements on quenched samples. ${ }^{43}$ The lines are fits using a Cole-Cole relaxation process eqn (1) and an Arrhenius dependence of the mean relaxation time, yielding $E_{\mathrm{a}}=0.47 \pm 0.06 \mathrm{eV}, \tau_{0}=1.6 \times 10^{-15} \mathrm{~s}$, $Y_{\infty}=36 \mathrm{GPa}, \Delta Y=18.3 \pm 0.2 \mathrm{GPa}$ and $\alpha=0.28 \pm 0.01$

above P1 are visible, but also $Y^{\prime \prime}$ can be well fitted for a considerable temperature range around the peak maximum.

The same quality of the fits has been found for G5 (Fig. 4) and V10 (Fig. 5). The agreement with the fits from dielectric data $^{48}$ of confined water is remarkable. E.g. Table 1 of ref. 48 shows fit parameters for dielectric measurements of water confined in MCM-41 with pore diameters of $2.1 \mathrm{~nm}$ (C10) and $3.6 \mathrm{~nm}$ (C18). Our P1 should be identified with their process 2, which for C10 $(2.1 \mathrm{~nm})$ yielded an activation energy (for $T \leq 170 \mathrm{~K})$ of $E_{\mathrm{a}}=0.47-0.49 \mathrm{eV}$ with $\tau_{0}=2 \times 10^{-18} \mathrm{~s}$ and a broadening parameter $\alpha=0.45-0.48$. Our values are nearly identical. Only our broadening parameter is a little smaller, which is not surprising, since Vycor and Gelsil have a broader pore size distribution compared to MCM-41.

To obtain the temperature dependence of the corresponding relaxation time, we used two different procedures, i.e. based on a simple analysis of the peak shifts with frequency and by fitting the data with a Cole-Cole relaxation function eqn (1). Since the ColeCole relaxation is symmetric, the mean relaxation time $\tau$ of eqn (1) can be determined either from fitting the data with eqn (1), or from the inverse of the peak angular frequency $\omega_{\mathrm{P}}{ }^{-1}$ of $Y^{\prime \prime}$.

With $\tau_{\mathrm{P}_{1}}=\tau_{01} \exp \left(E_{\mathrm{a}} / k_{\mathrm{B}} T\right)$ and $f_{\mathrm{P}_{1}}=1 / 2 \pi \tau_{\mathrm{P}_{1}}$ we obtain from the corresponding Arrhenius plot (inset of Fig. 3) an activation 

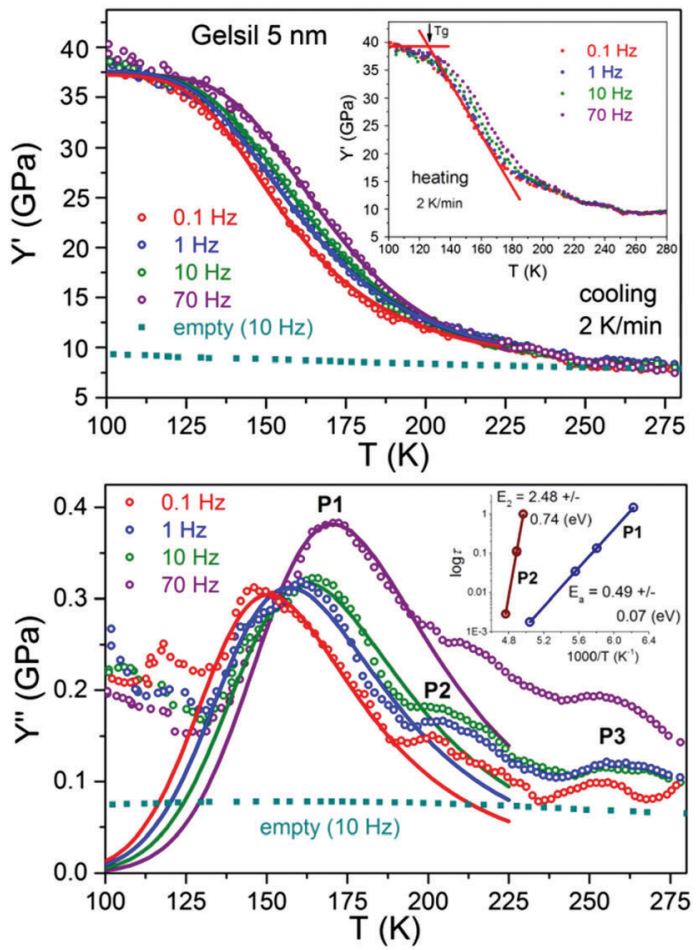

Fig. 4 Temperature dependencies of $Y^{\prime}$ and $Y^{\prime \prime}$ of slowly cooled water in Gelsil $5 \mathrm{~nm}$ measured at various frequencies. The blue squares show results of empty samples. The insets show heating runs at various frequencies and an Arrhenius plot corresponding to P1 and P2. The lines are fits using a Cole-Cole relaxation process eqn (1) and an Arrhenius dependence of the mean relaxation time, yielding $E_{\mathrm{a}}=0.49 \pm 0.07 \mathrm{eV}$, $\tau_{0}=3.8 \times 10^{-18} \mathrm{~s}, Y_{\infty}=37.5 \mathrm{GPa}, \Delta Y=27 \pm 0.3 \mathrm{GPa}$ and $\alpha=0.22 \pm 0.01$

energy of $E_{\mathrm{a}}=0.47 \pm 0.06 \mathrm{eV}$. Thus P1 can be identified with the low temperature main dielectric relaxation of confined water which also shows Arrhenius behaviour with the same activation energy, regardless of the type of confinement (see e.g. Fig. 1 of ref. 7).

Fig. 4 and 5 show cooling/heating runs at $2 \mathrm{~K} \mathrm{~min}^{-1}$ at various frequencies for water confined in Gelsil $5 \mathrm{~nm}$ and Vycor $10 \mathrm{~nm}$, respectively, and corresponding fits with eqn (1), yielding increasing activation energies with increasing pore size.

Inspecting Fig. 2-5 we find that the main difference between the quenching experiments ${ }^{43}$ and the slow cooling/heating runs occurs in $Y^{\prime}$ in the temperature region between $\approx 180 \mathrm{~K}$ and the freezing/melting transition. However, the region of the glass transition is not influenced by the different cooling rates. From the frequency dependence of P1 we obtain the same activation energies around $0.5 \mathrm{eV}$ and the same pore size dependence of P1 as for the quenched case.

Although the present data are very well reproducible and meaningful, due to the complexity of the problem interpretation of the data is not unambiguous and it is instructive to discuss them in the light of previous experiments and interpretations.

\section{Comparison with adiabatic calorimetry}

The glass transition temperature $T_{\mathrm{g}}$ is usually defined from differential scanning calorimetry (DSC) measurements by the onset of the heat capacity rise, which corresponds to the onset
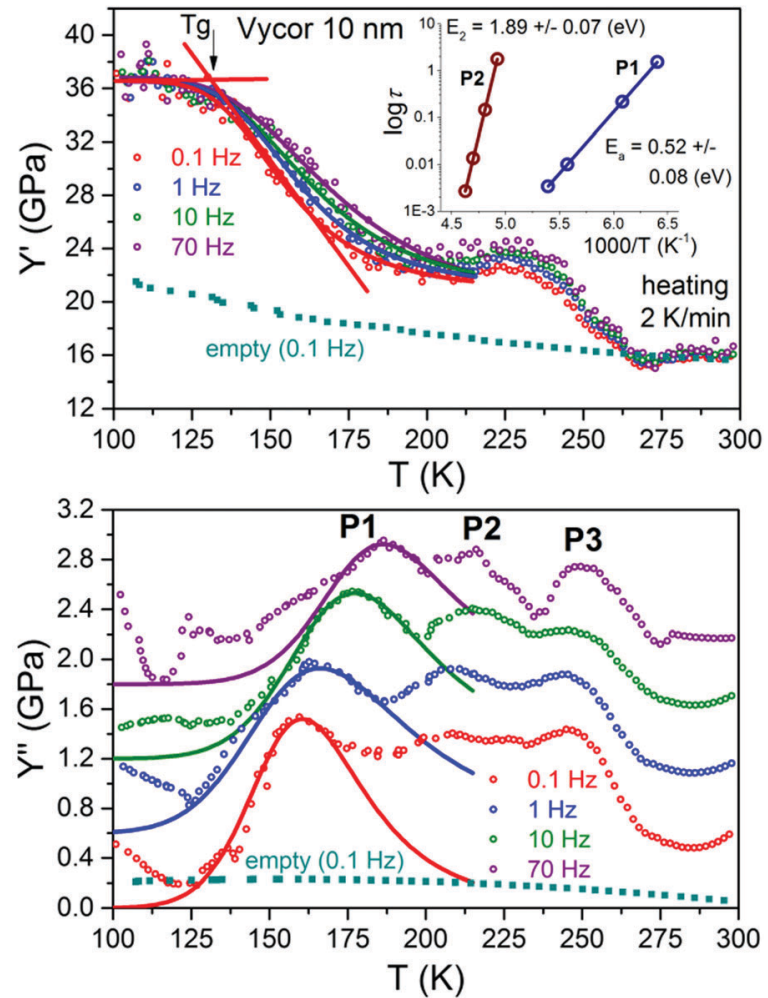

Fig. 5 Temperature dependencies of $Y^{\prime}$ and $Y^{\prime \prime}$ of slowly cooled water at heating with a rate of $2 \mathrm{~K} \mathrm{~min}^{-1}$ in Vycor $10 \mathrm{~nm}$ measured at various frequencies. Note that in $Y^{\prime \prime}$ the curves for 1,10 and $70 \mathrm{~Hz}$ are shifted from the original $0.1 \mathrm{~Hz}$ data for clarity. The blue squares show results of empty samples. The inset shows Arrhenius plots corresponding to P1 and P2. The lines are fits using a Cole-Cole relaxation process eqn (1) and an Arrhenius dependence of the mean relaxation time, yielding $E_{a}=0.52 \pm 0.08 \mathrm{eV}$, $\tau_{0}=3 \times 10^{-19} \mathrm{~s}, Y_{\infty}=36.3 \mathrm{GPa}, \Delta Y=14.2 \pm 0.2 \mathrm{GPa}$ and $\alpha=0.2 \pm 0.02$.

of the decay in Young's modulus $Y^{\prime \prime}$ (see insets in Fig. 3-5). However, if the jump in heat capacity around $T_{\mathrm{g}}$ is small - as it is the case for water ${ }^{50}$ - other methods are more appropriate. Adiabatic calorimetry turned out to be a very powerful tool for the identification of glass transition temperatures of confined water.

A comparison of our data with adiabatic calorimentry ${ }^{8-11}$ of water in various nanoporous systems in addition to the comparison with dielectric studies ${ }^{7,12,18,48}$ turns out to be very helpful for a discussion of the behaviour of water in nanopores. Oguni et al. ${ }^{8-11}$ measured the enthalpy relaxation $\mathrm{d} H / \mathrm{d} t$ of water in two different confining systems. They used silica-gels ${ }^{8,9}$ with rather irregular pore structures and broad pore size distributions with average diameters $\dagger$ of $3,6,12$ and $52 \mathrm{~nm}$ and MCM-41 ${ }^{10,11}$ having regular cylindrical pores with diameters between 1.5 and $5 \mathrm{~nm}$. The technique can be considered as a very low frequency spectroscopic method, ${ }^{51}$ where timescales of relaxation can be detected between $10^{2} \mathrm{~s}$ and $10^{6} \mathrm{~s}$. The glass transition temperature is then determined at the point at which $-\mathrm{d} H / \mathrm{d} t$ changes sign (for rapidly cooled

$\dagger$ There is some confusion in the pore sizes presented in various papers of Oguni et al. In ref. 8, Fig. 6a the pore diameter is given as $1.1 \mathrm{~nm}$. Exactly the same curve can be found in ref. 9 with a pore diameter of $3 \mathrm{~nm}$. According to the present DMA data the pore diameter of Fig. $6 \mathrm{a}$ in ref. 8 is most likely $3 \mathrm{~nm}$. 


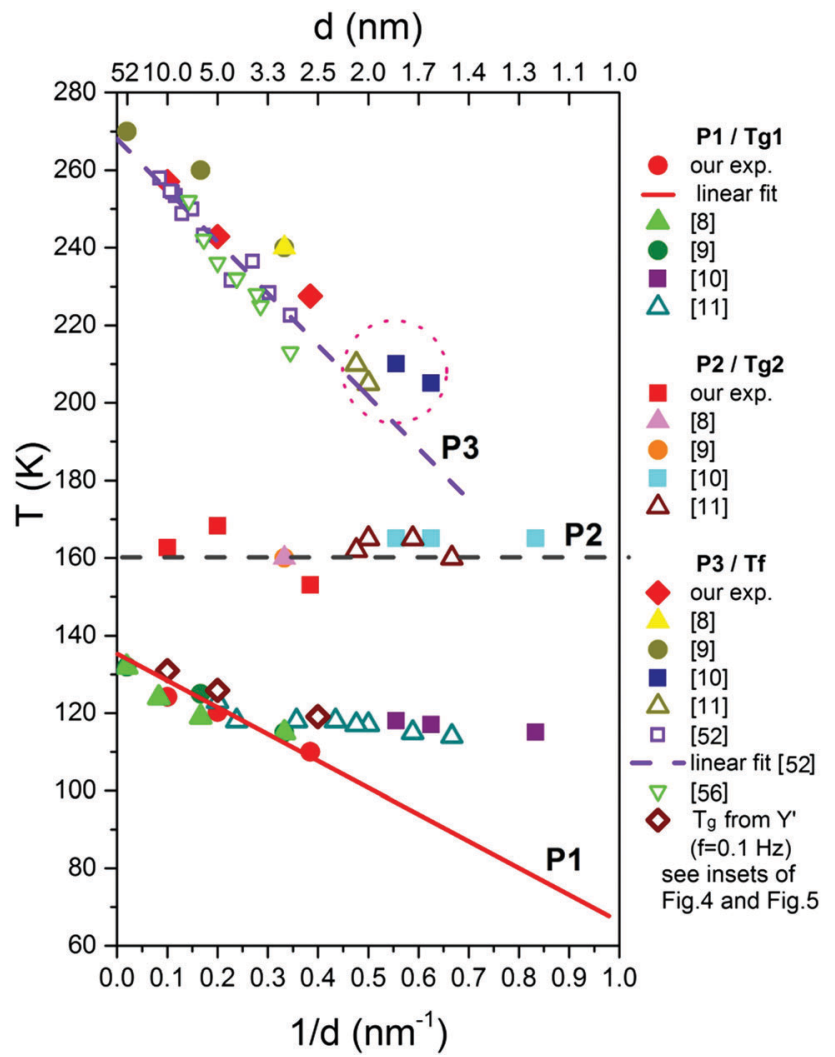

Fig. 6 Pore size dependencies of the processes P1, P2 and P3 from DMA measurements and adiabatic calorimetry data. ${ }^{8-11}$

samples) or shows a minimum (for slowly cooled samples). The so determined $T_{\mathrm{g}}$ value corresponds ${ }^{8}$ to a relaxation time $\tau=10^{3} \mathrm{~s}$.

Very similar to our results, the authors observed up to three peaks, which we identify with our three peaks P1, P2 and P3, observed in $Y^{\prime \prime}$ as follows (Fig. 6):

P3 of our data results from the freezing/melting process of water/ice in the core of the pores. This can be verified e.g. by the observed downshift of P3 with decreasing pore size (in agreement with ref. 52 and 56), the observed anomalies in thermal expansion (ref. 43 and Fig. 1 of the present work) and the fact that P3 does not shift with frequency, as expected for a (first order) freezing/melting phase transition.

The processes $\mathrm{P} 1$ and $\mathrm{P} 2$ are also observed in adiabatic calorimetry. ${ }^{8-11}$ Our frequency dependent measurements (Fig. 3-5) show that both processes are thermally activated. As already mentioned above, the process $\mathrm{P} 1$, which for $d<2.5 \mathrm{~nm}$ occurs around $118 \mathrm{~K}$, corresponds to the main ("universal") dielectric relaxation of confined water, which by many authors $^{12}$ is identified with the glass-to-liquid transition of supercooled water.

The frequency shift of $\mathrm{P} 2$ can also be fitted with an Arrhenius dependence of the relaxation time, i.e. $\tau_{\mathrm{P}_{2}}=\tau_{02} \exp \left(E_{2} / k_{\mathrm{B}} T\right)$, but yields a much higher activation energy $E_{2} \approx 2-2.5 \mathrm{eV}$ (Fig. 4 and 5) compared to P1. Moreover, its position is independent of pore size.

Extrapolating the relaxation times $\tau_{\mathrm{Pi}}$ to $10^{3} \mathrm{~s}$, we find that our $\mathrm{P} 1$ corresponds to the lower temperature peak (faster process) of $-\mathrm{d} H / \mathrm{d} t$, whereas $\mathrm{P} 2$ can be identified with the higher temperature peak (slower process) at $\approx 160 \mathrm{~K}$ (Fig. 6) of the adiabatic calorimetry data. ${ }^{8-11}$ This identification is not obvious from a first glance, because all peak positions depend strongly on the measurement time scale and the extrapolation to $10^{3} \mathrm{~s}$ shifts our peaks P1 and P2 to lower temperatures.

In this way we find - by including the data of adiabatic calorimetry - a very interesting systematic behaviour (Fig. 6) of the processes P1, P2 and P3 with varying pore size.

As Fig. 6 shows, P3 definitely corresponds to the freezing/ melting process of internal water/ice in nanopores, i.e. the slope $\mathrm{d} T_{\mathrm{f}} / \mathrm{d}(1 / d) \approx 110 \mathrm{~K} \mathrm{~nm}$ fits well with previous findings. ${ }^{49,52}$ For pore sizes $d>2.5 \mathrm{~nm}$ also Oguni et al. identified this process (P3) with freezing/fusion of internal water/ice in the pores. But for pore sizes below $c a .2 .5 \mathrm{~nm}$, Oguni et al. ${ }^{10,11}$ found three broad peaks in $-\mathrm{d} H / \mathrm{d} t$, where the high temperature peak around $210 \mathrm{~K}$ led them to the conclusion that $T_{\mathrm{g}}=210 \mathrm{~K}$ for bulk water. Inspecting Fig. 6, we strongly doubt this conclusion. Since these peaks around $210 \mathrm{~K}$ (see encircled data points of Fig. 6) are located at the extrapolated freezing line $T_{\mathrm{f}}(d)$, we think that the observed peak around $210 \mathrm{~K}$ results from freezing of water in small pores rather than a glass transition. Moreover, combining the available data of the two other processes, we find that P2 (extrapolated to $10^{3} \mathrm{~s}$ ) of our DMA measurements corresponds to the calorimetric anomaly observed at $\approx 160 \mathrm{~K}$ (Fig. 6).

Oguni et al. related their higher $T$ process $\mathrm{P} 2$ around $160 \mathrm{~K}$ to the effect of glass freezing of "internal water" (water molecules surrounded only by water molecules inside the pores) in the pores, and the process P1 at lower temperature to "interfacial water", whose molecules are bonded to the silanol groups of pore walls. Based on this interpretation, they concluded ${ }^{8}$ that the glass transition temperature $T_{\mathrm{g}}$ of bulk water is around $160 \mathrm{~K}$ or above and later they suggested ${ }^{10,11} T_{\mathrm{g}}$ of bulk water is even around $210 \mathrm{~K}$, as already mentioned above.

However, inspecting Fig. 6, it is obvious that Oguni's data have to be reinterpreted: as already mentioned above, it is most likely that the data of Oguni et al. around $210 \mathrm{~K}$ (encircled data in Fig. 6) correspond to the freezing/melting process of internal water in small pores and are not related to the glass transition of water.

Moreover, based on the present data and recent computer simulations, ${ }^{53-55}$ showing that the dynamics of water molecules is dramatically slowed down with decreasing distance to the pore walls, we interpret the processes corresponding to P1 and P2 quite opposite to Oguni et al. We claim that P2 (at about $160 \mathrm{~K}$ ) whose corresponding $\tau$ has a much higher activation energy (ca. $2 \mathrm{eV}$ ) than P1 (ca. $0.5 \mathrm{eV}$ ) - corresponds to a relaxation dynamics of water molecules which are bound to silanol groups of pore walls and therefore considerably slowed down compared to water molecules away from the pore walls which are surrounded by other water molecules and thus experiencing much faster dynamics. ${ }^{53-55}$ It should be noted that Ngai [ref. 12, p. 470] came to the same conclusion. However, he argued - in agreement with other authors - that the process P1 around $115 \mathrm{~K}$, which is caused by the relaxation of internal water, is not sensitive to the form of confinement or mixtures. Inspecting Fig. 6, one clearly observes that this is in fact the case only for pore sizes up to 
about $2.5 \mathrm{~nm}$. For pore sizes above $2.5 \mathrm{~nm}$ a systematic pore size dependence of $T_{\mathrm{g}} \sim T_{\mathrm{g}}^{\infty}-1 / d$ is found, which extrapolates to $T_{\mathrm{g}}^{\infty} \approx 136 \mathrm{~K}$ for $d \rightarrow \infty$.

\section{Comparison with dielectric spectroscopy and neutron scattering}

Broadband dielectric spectroscopy was intensively used to study the dynamics of supercooled water in nanopores as well as in aqueous solutions. Ample literature can be found e.g. in ref. 1 and 13. An important finding of these experiments is that around $T_{\text {cross }} \approx 190 \pm 20 \mathrm{~K}$ a dynamic crossover $^{1,13,20}$ of the water dynamics of supercooled confined water from Arrhenius ( $\beta$-process for $T<T_{\text {cross }}$ ) to Vogel-Fulcher ( $\alpha$-process for $T>T_{\text {cross }}$ ) behaviour is observed in dielectric spectroscopy and in neutron scattering (QENS). Most QENS studies ${ }^{19,20}$ of supercooled water locate this crossover at a temperature $T_{\text {cross }}$ of about 225 K. Despite an enormous amount of work, the physical origin of this dynamic crossover is not yet clarified. ${ }^{13}$ Some possible interpretations including the assumption of a "fragileto-strong transition" have already been discussed in Section 1. Many of the proposed scenarios (freezing in the tail of a notcompleted order-disorder transition, freezing of dipole reorientation dynamics, etc.) imply that water is macroscopically solid below $T_{\text {cross. }}$. Due to the restricted frequency range of the DMAmethod $(0.1-100 \mathrm{~Hz})$ we can follow the main relaxation of water in nanopores only at temperatures below $c a .200 \mathrm{~K}$, where the dynamics shows Arrhenius behaviour and we cannot access the change in dynamics up to the super-Arrhenius regime. However, we think that any interpretation which involves water behaving as a macroscopic solid below $T_{\text {cross }}$ is in contradiction with our data for the following reason: inspecting Fig. 3-5 one observes that the real part $Y^{\prime}$ of the complex Young's modulus decreases nearly to the background value of the silica matrix/water composite at about $225 \mathrm{~K}$, implying that water in the pores cannot be solid just below $T_{\text {cross. }}$. Moreover, it is hard to imagine that freezing of a local $\beta$-process can lead to such a big change in Young's modulus (see Section 4 for further discussion).

Another proposed interpretation relates the observed $\alpha \rightarrow \beta$ crossover of water in confinement to a finite size effect. Meanwhile, it is well established ${ }^{34,57,58}$ that glass transitions are accompanied by a growing length scale $\xi \sim \xi_{0} /\left(T-T_{\mathrm{VF}}\right)^{\gamma}$ of dynamically correlated regions. The idea is that the vanishing of the $\alpha$-relaxation and the related crossover appears to be due to the fact that the size $\xi$ of dynamically correlated regions reaches the confinement size $d$ at $T_{\text {cross }}$, i.e. $\xi\left(T_{\text {cross }}\right)=d$. This implies that below $T_{\text {cross }}$ no correlated motion over a distance larger than $d$ is possible and as a result the high-temperature VFT dependence of $\tau=\tau_{0} \exp \left[\xi^{3} \Delta / k_{\mathrm{B}} T\right]=\tau_{0} \exp \left[\xi_{0}{ }^{3} \Delta /(T-\right.$ $\left.\left.T_{\mathrm{VF}}\right)^{3 \gamma} k_{\mathrm{B}} T\right]$ transforms naturally to a low-temperature Arrhenius behavior $\tau=\tau_{0} \exp \left[d^{3} \Delta / k_{\mathrm{B}} T\right]$. This scenario would imply that the dynamic correlation length $\xi$ of water should be in the range of a few nanometers around $T_{\text {cross }} \approx 200 \mathrm{~K}$.

Such an interpretation of the observed $\alpha-\beta$ crossover would be consistent with our present data, since it explains the liquid like behaviour of the Young's modulus extending to temperatures much below $200 \mathrm{~K}$, despite the fact that the $\alpha$-relaxation disappears. In this picture, the Arrhenius behaviour occurs due to a finite size effect (the cooperative motion cannot grow larger than the pore size) and not due to the fact that the motion is local. For water there exist no data about the size of dynamical correlations, but values of $\xi \approx 1-3 \mathrm{~nm}$ in the vicinity of $T_{\mathrm{g}}$ have been reported ${ }^{34,59,60}$ for a number of other glass forming liquids.

\section{Summary and conclusions}

In the present work we have performed extensive dynamical mechanical measurements of water confined in nanoporous silica of various pore sizes $(2.5,5$ and $10 \mathrm{~nm})$, with heating and cooling at a rate of $2 \mathrm{~K} \mathrm{~min}^{-1}$ and at frequencies between $0.1 \mathrm{~Hz}$ and $70 \mathrm{~Hz}$. The results are in excellent agreement with earlier DMR (dynamic mechanical resonance) measurements ${ }^{44}$ which have been performed on water confined in large bars $(200 \times 8 \times$ $4.4 \mathrm{~mm}^{3}$ ) of Vycor samples, vibrating in free mode at a frequency of about $400 \mathrm{~Hz}$. Due to the higher measurement frequency the authors find a $\tan \delta$ peak (corresponding to our P1-peak in $Y^{\prime \prime}$ ) at a higher temperature (i.e. at $188 \mathrm{~K}$, Fig. 5 of ref. 44), in perfect agreement with the Arrhenius dependence of the relaxation time with $E_{\mathrm{a}} \approx 0.5 \mathrm{eV}$. Quite similar to our data, these authors measured also a weakly increasing Young's modulus with decreasing temperature due to freezing of water in the core of the pores (which they call the "capillary transition") and a much stronger increase with decreasing temperature in the vicinity of the P1 peak. In a quite consistent explanation, the authors assume that ice forms in the centre of the pores and a film (a few layers) of adsorbed water remains liquid and separates the ice from the silicon matrix. Such a core-shell ice-water structure in nanoporous systems is also confirmed by $\mathrm{NMR}^{47}$ and $\mathrm{THz}$ optical spectroscopy, ${ }^{46}$ etc.

In this scenario, decreasing temperature leads to an increase in the viscosity of the adsorbed film, until at very low temperatures (around P1) the adsorbed water layer freezes and effectively cements the silicon matrix and the ice together. We think that this model is very convincing and explains also the main body of our measurements. As already mentioned above, most authors agree that not all water crystallizes to ice ${ }^{47,61}$ in nanoporous systems, but a film of a few layers remains liquid down to very low temperature.

However, some discrepancy appears in the interpretation of the increase in the viscosity of the adsorbed film with decreasing temperature. Many researchers (including ourselves) attribute the freezing transition around P1 of the adsorbed water to the glass transition of confined supercooled water. But already in ref. 62 the authors follow an opposite string of arguments. Based on mechanical relaxation measurements ${ }^{63}$ of pure and doped ice, the authors interpret the observed relaxation (with $E_{\mathrm{a}} \approx 0.5 \mathrm{eV}$ ) due to proton movement at lattice defects of the Bjerrum ${ }^{64}$ type. Very recently, Shephard and Salzmann ${ }^{30}$ came up with a similar interpretation. They state that the anomalies of amorphous ices which are usually interpreted as glass to liquid transitions - are in fact due to unfreezing of dipole reorientations. The real glass-toliquid transition would then occur at higher temperature, where long range diffusion of water molecules sets in. 
We think that such a scenario ${ }^{30}$ of unfreezing of the dipole reorientation dynamics upon heating can be hardly ruled out. Especially if one takes into account the similarities of activation energies ${ }^{65}$ between ice $\left(E_{\mathrm{a}}=0.57 \mathrm{eV}\right.$, determined by mechanical and dielectric relaxation measurements) and confined supercooled water (see e.g. Fig. 3-5 or ref. 7 and 12). However, the mechanical measurements ${ }^{63}$ on ice have shown that the strain produced by proton reorientation is small and does not produce large changes in the elastic constants. This is a rather general result for orientationally active materials, e.g. we have previously measured similar effects ${ }^{29}$ in $\mathrm{C}_{60}$, where we have detected a pronounced $\tan \delta$ peak in the vicinity of the orientational glass transition, accompanied by small changes in Young's modulus. Thus we believe that the observed large changes in Young's modulus $Y^{\prime}$ (Fig. 2-5, and ref. 44 and 62) accompanying the relaxation peak $\mathrm{P} 1$ in $Y^{\prime \prime}$ are caused by a glass-to-liquid transition in the supercooled film of adsorbed water due to unfreezing of translational diffusion, rather than by reorientation dynamics of water molecules.

Let us state it in another way: if the large softening detected previously $^{44,62}$ and in the present work in Young's modulus (Fig. 3-5) was due to unfreezing of proton reorientations and not to long range diffusion, the adsorbed water film should have some pseudo-crystalline structure with an extremely small Young's modulus. Frankly speaking this would be a very unusual material behaviour. We think that the elastic measurements here are more exploratory in comparison to dielectric measurements, where the polarization produced by bond orientation in an electric field is large and thus cannot be easily used to discriminate between a liquid and a solid material.

If we accept that the relaxation around $\mathrm{P} 1$ originates from a glass-to-liquid transition of supercooled water, we can draw the following conclusions:

Extrapolating our data to the timescale $\left(10^{3} \mathrm{~s}\right)$ of adiabatic calorimetry, we obtain the following systematic behaviour (Fig. 6) of water anomalies with varying pore size.

- The anomaly around $160 \mathrm{~K}$ is independent of pore size. It results most probably from freezing of "interfacial water", i.e. water molecules that are bound to the surface of the pore walls.

- The anomaly around $115 \mathrm{~K}$ (for pore sizes below $2.5 \mathrm{~nm}$ ) results from glass freezing of water molecules that are surrounded by other water molecules. This process - which corresponds to the "universal" relaxation of confined supercooled water - is independent of pore size only for pore sizes below ca. $2.5 \mathrm{~nm}$. Above $2.5 \mathrm{~nm}$ it becomes strongly dependent on pore size, i.e. $\Delta T_{\mathrm{g}}(d) \sim 1 / d$, leading to $T_{\mathrm{g}}(d=\infty) \approx 136 \mathrm{~K}$. As already mentioned above the pronounced softening of the real part of the complex Young's modulus (Fig. 3-5) starting in the vicinity of the $\mathrm{P}_{1}$ peak strongly supports the conjecture that the anomaly around $115-136 \mathrm{~K}$ corresponds to a transition from glass to liquid and not to a local $\beta$-relaxation.

We finally note that - since our measurements have been performed on water confined in nanopores - we cannot definitely conclude that the extrapolated (to $d \rightarrow \infty$ ) glass transition temperature is identical to $T_{\mathrm{g}}$ of bulk water. And in this sense, the question of $T_{\mathrm{g}}$ of bulk water still remains an open issue.

\section{Conflicts of interest}

There are no conflicts to declare.

\section{Acknowledgements}

We acknowledge financial support from the Austrian Science Fund (FWF) Grant No. P 28672-N36.

\section{Notes and references}

1 S. Cerveny, F. Mallamace, J. Swenson, M. Vogel and L. Xu, Chem. Rev., 2016, 116, 7608.

2 A. Hallbrucker, E. Mayer and G. P. Johari, J. Chem. Phys., 1989, 93, 4986.

3 G. P. Johari, A. Hallbrucker and E. Mayer, Nature, 1987, 330, 552.

4 I. Kohl, L. Backmann, A. Hallbrucker, E. Mayer and T. Loerting, Phys. Chem. Chem. Phys., 2005, 7, 3210.

5 V. Velikov, S. Borick and C. A. Angell, Science, 2001, 294, 2335. 6 Y. Yue and C. A. Angell, Nature, 2004, 427, 717.

7 S. Cerveny, G. A. Schwartz, R. Bergman and J. Swenson, Phys. Rev. Lett., 2004, 93, 245702.

8 M. Oguni, S. Maruyama, K. Wakabayashi and A. Nagoe, Chem. - Asian J., 2007, 2, 514.

9 S. Maruyama, K. Wakabayashi and M. Oguni, in CP708 "Slow Dynamics in Complex Systems: 3rd International Symposium”, ed. M. Tokuyama and I. Oppenheim, 2004, p. 675.

10 M. Oguni, Y. Kanke and S. Namba, in CP982 "Complex Systems: 5th International Workshop on Complex Systems”, ed. M. Tokuyama, I. Oppenheim and H. Nishiyama, 2008, p. 34.

11 M. Oguni, Y. Kanke, A. Nagoe and S. Namba, J. Phys. Chem. B, 2011, 115, 14023.

12 K. L. Ngai, Relaxation and Diffusion in Complex Systems, Springer Science + Business Media, LLC, 2011, DOI: 10.1007/ 978-1-4419-7649-9.

13 J. Swenson and S. Cerveny, J. Phys.: Condens. Matter, 2015, 27, 033102.

14 G. P. Johari, J. Chem. Phys., 1996, 105, 7079.

15 K. Ito, C. G. Moynihan and C. A. Angell, Nature, 1999, 398, 492.

16 R. Bergman and J. Swenson, Nature, 2000, 403, 283.

17 J. Sjostrom, J. Swenson, R. Bergman and S. Kittaka, J. Chem. Phys., 2008, 128, 154503.

18 H. Jansson and J. Swenson, Eur. Phys. J., 2003, E12, S51.

19 F. Mallamace, M. Broccio, C. Corsaro, A. Faraone, U. Wanderlingh, L. Liu, C. Y. Mou and S. H. Chen, J. Chem. Phys., 2006, 124, 161102.

20 A. Faraone, L. Liu, C. Y. Mou, C. W. Yen and S. H. Chen, J. Chem. Phys., 2004, 121, 10843.

21 L. Xu, P. Kumar, S. V. Buldyrev, S. H. Chen, P. H. Poole, F. Sciortino and H. E. Stanley, Proc. Natl. Acad. Sci. U. S. A., 2005, 102, 16558.

22 F. Mallamace, C. Branca, C. Corsaro, N. Leone, J. Spooren, S.-H. Chen and H. E. Stanley, PNAS, 2010, 107, 22457.

23 A. Dehaoui, B. Issenmann and F. Caupin, PNAS, 2015, 112, 12020. 24 W. Götze and L. Sjogren, Rep. Prog. Phys., 1992, 55(3), 241. 25 R. J. Speedy and C. A. Angell, J. Chem. Phys., 1976, 65, 851. 26 C. A. Angell, Science, 2008, 319, 582. 
27 G. Adam and J. H. Gibbs, J. Chem. Phys., 1965, 43, 139.

28 P. Mondal, P. Lunkenheimer and A. Loidl, Z. Phys. B: Condens. Matter, 1996, 99, 527.

29 W. Schranz, A. Fuith, P. Dolinar, H. Warhanek, M. Haluska and H. Kuzmany, Phys. Rev. Lett., 1993, 71, 1561.

30 J. J. Shephard and C. G. Salzmann, J. Phys. Chem. Lett., 2016, 7, 2281.

31 P. Pissis, J. Laudat, D. Daoukaki and A. Kyritsis, J. Non-Cryst. Solids, 1994, 171, 201.

32 W. Schranz, M. R. Puica, J. Koppensteiner, H. Kabelka and A. V. Kityk, Europhys. Lett., 2007, 79, 36003.

33 J. Koppensteiner, W. Schranz and M. R. Puica, Phys. Rev. B: Condens. Matter Mater. Phys., 2008, 78, 054203.

34 J. Koppensteiner, W. Schranz and M. A. Carpenter, Phys. Rev. B: Condens. Matter Mater. Phys., 2010, 81, 024202.

$35 \mathrm{~J}$. Koppensteiner, The glass transition in nanoscale confinement probed by dynamic mechanical spectroscopy, $\mathrm{PhD}$ thesis, University of Vienna, 2009.

36 A. Ghaffar, Confinement induced structural changes of alkali metals in nanoporous systems, PhD thesis, University of Vienna, 2014.

37 P. Levitz, G. Ehret, S. K. Sinha and J. M. Drake, J. Chem. Phys., 1991, 95, 6151.

38 J.-L. R. Nogues and W. Moreshead, J. Non-Cryst. Solids, 1990, 121, 136.

39 N. Eschricht, E. Hoinkis, F. Mädler and P. Schubert-Bischoff, Stud. Surf. Sci. Catal., 2002, 144, 355.

40 S. Gruener, T. Hofmann, D. Wallacher, A. V. Kityk and P. Huber, Phys. Rev. E: Stat., Nonlinear, Soft Matter Phys., 2009, 79, 067301.

41 W. Schranz, Phase Transitions, 1997, 64, 103.

42 E. K. H. Salje and W. Schranz, Z. Kristallogr., 2011, 226, 1.

43 V. Soprunyuk, W. Schranz and P. Huber, Europhys. Lett., 2016, 115, 46001.

44 E. J. Sellevold and F. Radjy, J. Mater. Sci., 1976, 11, 1927.

45 A. K. Soper, Chem. Phys. Lett., 2013, 590, 1.
46 A. Taschin, P. Bartolini and R. Torre, Meas. Sci. Technol., 2017, 28, 014009.

47 Y. Fukatsu, K. Morikawa, Y. Ikeda and T. Tsukahara, Anal. Sci., 2017, 33, 903.

48 J. Sjöström, S. Swenson, R. Bergman and S. Kittaka, J. Chem. Phys., 2008, 128, 154503.

49 A. Schreiber, I. Ketelsen and G. H. Findenegg, Phys. Chem. Chem. Phys., 2001, 3, 1185.

50 C. A. Angell, Science, 2008, 319, 582.

51 H. Suga and S. Seki, Faraday Discuss. Chem. Soc., 1980, 69, 221.

52 G. H. Findenegg, S. Jähnert, D. Akcakayiran and A. Schreiber, ChemPhysChem, 2008, 9, 2651.

53 F. Klameth and M. Vogel, J. Phys. Chem. Lett., 2015, 6, 4385.

54 C. Allolio, F. Klameth, M. Vogel and D. Sebastiani, ChemPhysChem, 2014, 15, 3955.

55 F. Klameth and M. Vogel, J. Chem. Phys., 2013, 138, 134503. 56 K. Morishige and K. Kawano, J. Chem. Phys., 1999, 110, 4876. 57 S. Albert, Th. Bauer, M. Michl, G. Biroli, J.-P. Bouchoud, A. Loidl, P. Lunkenheimer, R. Tourbot, C. Wiertel-Gasquet and F. Ladieu, Science, 2016, 352, 1308.

58 Th. Bauer, P. Lunkenheimer and A. Loidl, Phys. Rev. Lett., 2013, 111, 225702.

59 E. Hempel, G. Hempel, A. Hensel, C. Schick and E. Donth, J. Phys. Chem. B, 2000, 104, 2460.

60 E. Donth, H. Huth and M. Beiner, J. Phys.: Condens. Matter, 2001, 13, L451.

61 R. T. Pearson and W. Derbyshire, J. Colloid Interface Sci., 1973, 46, 232.

62 F. Radjy and E. J. Sellevold, Nature, 1973, 241, 133.

63 D. Kuriowa, US Army Cold Regions Res. Eng. Lab., Res. Rep. 131, US Army Material Command, Hannover, 1965.

64 D. Eisenberg and W. Kauzmann, The Structure and Properties of Water, Oxford University Press, London, 1969, p. 116.

65 P. V. Hobbs, Ice Physics, Oxford University Press, London, 1974, p. 272. 